\title{
Rigid-Rod Polyesters with Flexible Side Chains IX. Phase Behavior Including Nematic, Layered, and Hexagonal Columnar Phases in Poly( $p$-biphenylene terephthalate) with Alkoxy Side Chains
}

\author{
Kai Fu, ${ }^{*}$ Nobuhiro SeKine, ${ }^{*}$ Masato Sone, ${ }^{*}$ Masatoshi ToKITA, ${ }^{*}$ \\ and Junji WATANABE ${ }^{*, * *, \dagger}$ \\ *Department of Polymer Chemistry, Tokyo Institute of Technology, Ookayama, Meguro-ku, \\ Tokyo 152-8552, Japan \\ ${ }^{* *}$ CREST-JST (Japan Science and Technology Corporation), 4-1-8 Hon-cho, Kawaguchi, \\ Saitama 332-0012, Japan
}

(Received January 11, 2002; Accepted March 26, 2002)

\begin{abstract}
In this study we prepared a series of poly( $p$-biphenylene terephthalate) with alkoxy side chains attached to terephthalate moiety. The carbon number of alkoxy side chains, $n$, is ranged from 8 to 18 . These polymers exhibited well-defined thermotropic phase behavior with a nematic phase in the higher temperature region and two ordered mesophases in the lower temperature region. One of the lower temperature mesophases was found to be a hexagonal columnar phase in the polymers with smaller values of $n$ ( 8 to 12), while the other is the layered mesophase preferentially formed from the polymer with $n=18$. In the polymers with $n=13,14$, and 16, the columnar and layered mesophases coexist. In these intermediate polymers, the relative amount of the layered phase to the columnar phase increases with the increase of $n$. Further, it increases when the mesophase temperature decreases, indicating that the layered phase is thermodynamically lower temperature mesophase than the columnar one. While the layered phase is occasionally observed in this kind of polymers with long alkyl side chains, the columnar phase is new and interesting since many chains are included in its hexagonal unit cell. The number of chains increases from 7 to 12 with an increase of $n$ from 8 to 14 . To achieve the reasonable packing of the aromatic main chains, we proposed the special hexagonal structure where the fully extended main chains are closely packed with lateral spacing of around $4.5 \AA$ to form a honeycombed network and the molten side chains are accommodated within a resulting cylindrical domain.
\end{abstract}

KEY WORDS Aromatic Polyesters / Alkyl Side Chain / Liquid Crystal / Hexagonal Columnar Phase / Layered Phase /

There have been several recent reports in the literature on thermotropic phase behavior of the rigid-rod polymers with long flexible alkyl side chains. ${ }^{1-16}$ The most interesting property of these materials is an ability to form layered structures in crystals and liquid crystals when the alkyl side chains reach a critical length. The layered mesophases are characterized by a closed packing of the rigid-rod main chains into layers with the molten side chains occupying a space between the layers. The driving force for an adoption of such a layered structure is a type of microphase separation of the aliphatic and aromatic domains, ${ }^{2,5,6,12}$ and the liquid crystallinity is the results of a partial or total lack of positional order with respect to the main-chain packing within a layer and of the molten side chains between the layers. ${ }^{12,13}$

In a previous paper, ${ }^{17}$ we studied the thermotropic phase behavior and structures of the rigid-rod $\operatorname{poly}(p$ biphenylene terephthalate) main chain with flexible dodecyloxy side chains attached to terephthalate moiety, It is interesting that this material forms a hexagonal

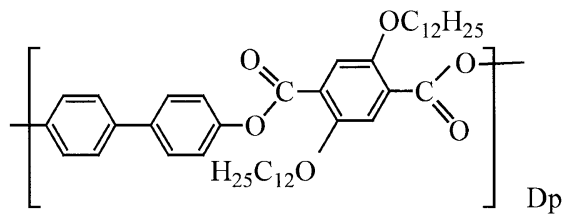

PBpT-012

columnar phase. This is the first example of a hexagonal columnar phase in this type of aromatic polyesters with flexible side chains. Although several examples of the columnar phase have so far been reported in the polymeric system, ${ }^{1,18-21}$ the columnar phase observed is especially interesting because approximately 9 to 10 molecules are included in a hexagonal lattice. Such a large number indicates that there exists a specific association of molecules. As the side chains are still conformationally disordered in this phase, a microsegregated structure can be visualized in which the aromatic main chains closely associate with each other to form a layer and the side chains stick out of both sides of layer. $^{2,5,6,12}$ In order to satisfy the condition that all the side chains are spaced with an equivalent environ-

${ }^{\dagger}$ To whom correspondence should be addressed (Phone: +81-3-5734-2633, Fax: +81-3-5734-2888, E-mail: jwatanab@polymer.titech.ac.jp). 
Table I. Characterization of PBp-On polyesters

\begin{tabular}{|c|c|c|c|c|c|c|}
\hline \multirow{2}{*}{ Sample } & \multicolumn{3}{|c|}{ GPC data } & \multirow{2}{*}{$\eta_{\mathrm{inh}} / \mathrm{dL} \mathrm{g}^{-1}$} & \multicolumn{2}{|c|}{ Transition temperatures } \\
\hline & $M_{\mathrm{n}}\left(\times 10^{4}\right)$ & $M_{\mathrm{w}}\left(\times 10^{4}\right)$ & $M_{\mathrm{w}} / M_{\mathrm{n}}$ & & $T_{1} /{ }^{\circ} \mathrm{C}$ & $T_{2} /{ }^{\circ} \mathrm{C}$ \\
\hline PBpT-O8 & 1.03 & 2.00 & 1.9 & $-^{\mathrm{a}}$ & 193 & 280 \\
\hline PBpT-O9 & 1.00 & 1.90 & 1.9 & $-^{\mathrm{a}}$ & 173 & 257 \\
\hline PBpT-O10 & 1.34 & 2.57 & 1.9 & 0.47 & 169 & 243 \\
\hline PBpT-O12 & 1.26 & 2.59 & 2.1 & 0.44 & 155 & 234 \\
\hline PBpT-O13 & 1.21 & 2.59 & 2.2 & 0.42 & 156 & 239 \\
\hline PBpT-O14 & 0.87 & 1.72 & 2.0 & 0.28 & 141 & 198 \\
\hline PBpT-O16 & 1.03 & 2.04 & 2.0 & 0.27 & 137 & 193 \\
\hline PBpT-O18 & 0.80 & 1.63 & 2.0 & 0.24 & 145 & 176 \\
\hline
\end{tabular}

${ }^{\mathrm{a}}$ The measurement were not done because of the poor solubility in THF.

ment and the main chains are packed with a reasonable lateral distance, we have supposed a distinct hexagonal packing structure where the aromatic main chains form the honeycombed network to surround the cylindrical side-chain domains.

In this study, the further examination on the thermotropic phase behavior including the columnar liquid crystal was performed by preparing a homologous series of polymers with alkoxy side chains in various lengths,

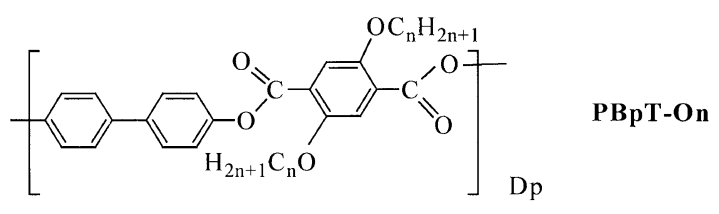

$\mathrm{n}=8,9,10,12,13,14,16,18$

These polymers were designated as PBpT-On where $n$ ranging from 8 to 18 indicates the carbon number of alkoxy side chain.

\section{EXPERIMENTAL}

\section{Materials}

PBpT-On polymers were synthesized by polycondensation of 4,4'-dihydroxybiphenyl with the 2,5bis(alkoxy)terephthaloyl chloride in dry pyridin at $60^{\circ} \mathrm{C}$. The inherent viscosity was measured in THF solutions at a concentration of $0.5 \mathrm{~g} \mathrm{dL}^{-1}$. The molecular weights were determined by gel permeation chromatography (GPC) with a column of Shodex K-2004, in chloroform at $40^{\circ} \mathrm{C}$ and with a calibration by polystyrene standards.

\section{Methods}

Differential scanning calorimetric (DSC) measurement was performed with a Perkin-Elmer DSC-II at a

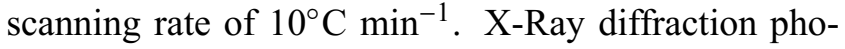
tographs were taken using a Rigaku-Denki X-Ray generator with Ni-filtered $\mathrm{Cu}-\mathrm{K} \alpha$ radiation. The sample temperatures were measured and regulated within an accuracy of $0.2^{\circ} \mathrm{C}$ by using a Mettler FP 80 heater. The film to specimen distance was determined by calibration with silicon powder.

\section{RESULTS}

\section{Thermal Behavior}

In order to eliminate any thermal prehistory, the sample was first heated to the isotropic melt and the reproducible DSC data were collected on cooling and heating. Two small transitions were observed for all the materials prepared here, and their transition temperatures, $T_{1}$ and $T_{2}$, are listed in Table I. The higher temperature transition at $T_{2}$ corresponds to the isotropicnematic phase transition while the lower one at $T_{1}$ corresponds to the transition from the nematic to some ordered mesophase. Transition enthalpies are fairly small for both. They are around $0.1-0.3 \mathrm{kcal} \mathrm{mol}^{-1}$. On normal cooling rate, no crystallization takes place from the mesophases. Transition temperatures are plotted against $n$ in Figure 1. Both temperatures decrease with the increase of $n$ from 8 to 18 .

\section{Mesophase Structure}

The higher temperature mesophase is obviously a nematic from the microscopic observation of schlierene texture. On cooling to the lower temperature mesophase, the texture becomes fine and the viscosity increases as well although it is still fluid when the shear is applied. ${ }^{17}$

The structural features of the lower temperature mesophases were analyzed by X-Ray diffraction method. Typical X-Ray patterns of the oriented fibers are shown in Figures 2a, 2b, and 2c for PBpT-O12, PBpT-O14, and PBpT-O18, respectively. Here, the oriented fiber was prepared by pulling the nematic melt and the fiber axis that corresponds to the chain axis is placed in the vertical direction. The basic spacing data are collected in Table II. At a glance of Figure 2, it is found that the type of mesophase is different between 
Table II. The X-Ray data of lower temperature mesophases ${ }^{\mathrm{a}}$

\begin{tabular}{|c|c|c|c|c|c|c|c|c|}
\hline & PBpT-O8 & PBpT-O9 & PBpT-O10 & PBpT-O12 & PBpT-O13 & PBpT-O14 & PBpT-O16 & PBpT-O18 \\
\hline \multicolumn{9}{|c|}{ [sharp inner reflections on the equatorial line] } \\
\hline $\mathrm{d}_{100}(\mathrm{HC})^{\mathrm{b}}$ & 17.7 & 19.3 & 20.6 & 23.4 & 25.2 & 26.4 & vw & \\
\hline $\mathrm{d}_{110}(\mathrm{HC})$ & 10.2 & 11.2 & 11.9 & 13.4 & 14.6 & & & \\
\hline $\mathrm{d}_{200}(\mathrm{HC})$ & 8.9 & & 10.3 & 11.7 & 12.6 & & & \\
\hline $\mathrm{d}_{210}(\mathrm{HC})$ & & & 7.8 & 8.9 & & & & \\
\hline $\mathrm{d}_{100}(\mathrm{~L})^{\mathrm{b}}$ & & & & & 19.3 & 20.1 & 22.1 & 24.2 \\
\hline $\mathrm{d}_{200}(\mathrm{~L})$ & & & & & & 10.1 & 11.2 & 12.1 \\
\hline $\mathrm{d}_{300}(\mathrm{~L})$ & & & & & & & 7.4 & 8.1 \\
\hline \multicolumn{9}{|c|}{ [outer broad reflections on the equatorial line] } \\
\hline $\mathrm{d}_{010}(\mathrm{HC})$ & 4.3 & 4.4 & 4.4 & 4.4 & 4.4 & $4.5^{\mathrm{c}}$ & $4.6^{\mathrm{c}}$ & $4.6^{\mathrm{c}}$ \\
\hline $\mathrm{d}_{010}(\mathrm{~L})$ & & & & & & 3.6 & 3.7 & 3.7 \\
\hline \multicolumn{9}{|c|}{ [meridional reflection] } \\
\hline $\mathrm{d}_{001}$ & 16.6 & 16.5 & 16.6 & 16.6 & 16.5 & 16.6 & 16.6 & 16.5 \\
\hline
\end{tabular}

${ }^{a}$ The X-Ray data were collected from as-spun fibers coold down to the room temperature after annealing at $100^{\circ} \mathrm{C}$ for $30 \mathrm{~min} .{ }^{\mathrm{b}} \mathrm{HC}$ : hexagonal columnar phase, $\mathrm{L}$ : layered phase. ${ }^{\mathrm{c}}$ The broad reflection that appears as ring.

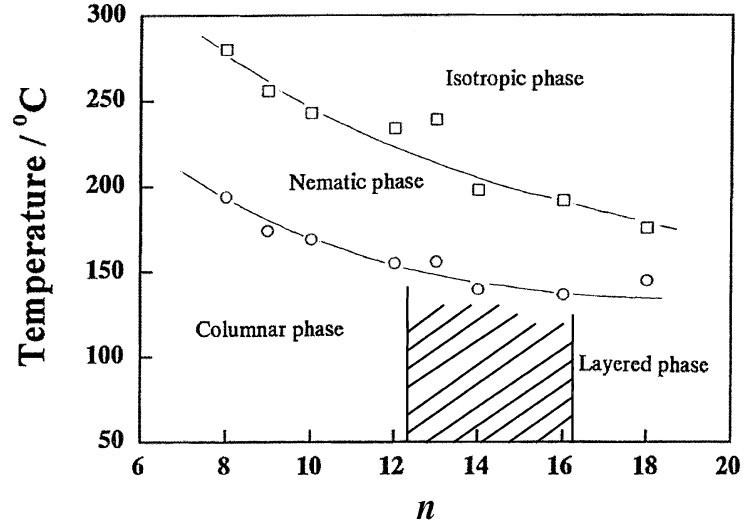

Figure 1. Variation of the transition temperatures with $n$, the number of carbon atoms in the alkoxy side chain. The transition temperatures are collected from the second heating DSC curves. The shaded area is the coexistence region of the columnar and layered phases (see the text).

the homologues with the smaller and larger values of $n$. The polymer with $n=12$ indicates the several sharp reflections on the equator, which can be indexed by a simple hexagonal packing in Table II. The broad outer reflection with the spacing of $4.4 \AA$ is also included. The similar pattern is observed in the polymers with $n=8$, 9 and 10 (see Table II). On the other hand, the polymer with the largest $n=18$ shows characteristic pattern including only the h00 reflections on an equatorial line (Figure 2c), which can be attributed to the layered structure. In this case, the broad outer reflection with a spacing of $3.7 \AA$ can be detected on the equatorial line. Both patterns also include the broad ring with a spacing of $4.4 \AA$ in the outer region, indicating that the side chains melt in these mesophases. The streak located on the meridional line shows the random displacement of the polymer chains along the chain axis, ${ }^{22}$ and its spac-

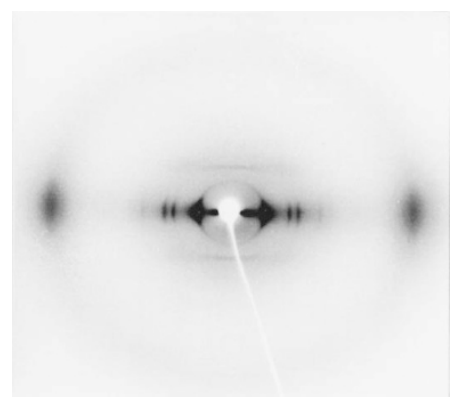

(a)

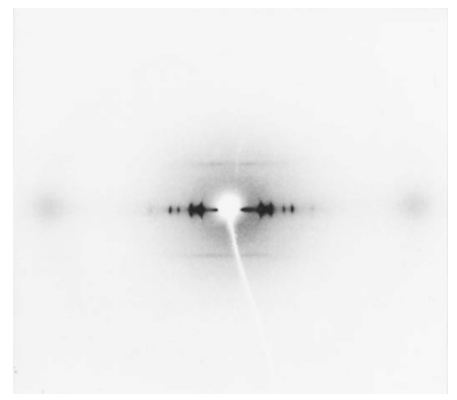

(b)

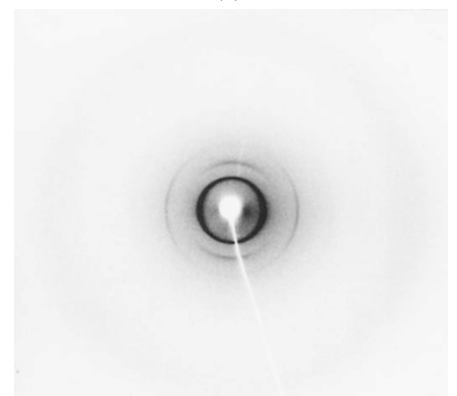

(c)

Figure 2. Typical X-Ray photographs of the low temperature mesophases observed for the fiber specimens of (a) PBpT-12, (b) PBpT-14, and (b) PBpT-18. Here, the oriented fiber was spun from the nematic melt and the fiber axis corresponding to the polymer chain axis is placed in the vertical direction. 


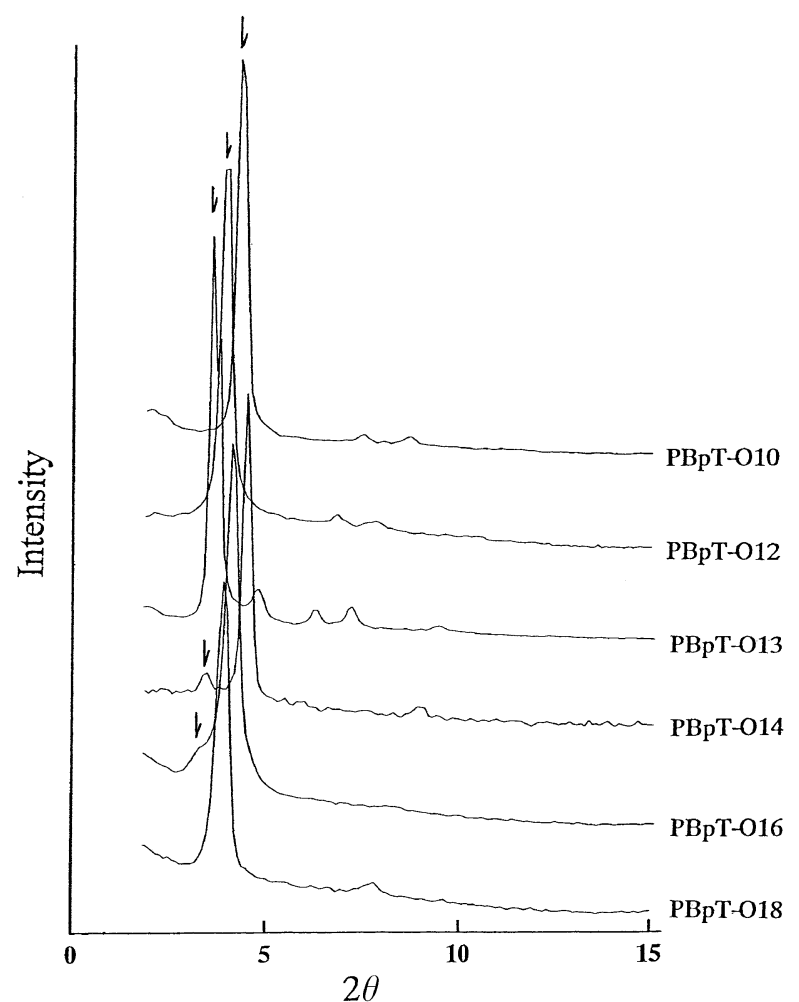

Figure 3. Intensity profile scanned along the equatorial line of oriented X-Ray patterns taken for the as-spun fibers from the nematic melt. The 100 reflections of the lower temperature mesophases are observed in the smaller angle region around $2 \theta=$ $3-5^{\circ}$. The 100 reflection of the columnar phase is differentiated by arrow from that of the layer phase.

ing of around $16.6 \AA$ shows that the polymer chains assume the extended conformation. ${ }^{12}$

In the polymers with intermediate $n$ of 13,14 , and 16 , the X-Ray pattern represents the coexistence of hexagonal and layered structures (see Figure 2b). More details can be found from the intensity profile scanned along the equatorial line of Figure 3. Here, the 100 reflections for the layered and columnar phases are observed in the small angle region around $2 \theta=4^{\circ}$. It clearly shows that the relative intensity of the 100 reflection of layered phase to that of hexagonal columnar one increases with an increase of $n$ from 13 to 16. In Figure 4, the spacings of the 100 reflections, $\mathrm{d}_{100}$, are plotted against $n$. The data points for each phase fall on well separated lines, clarifying that the polymers with smaller values of $n$ tend to form the hexagonal columnar mesophase while ones with larger values of $n$ tend to form the layered mesophase. An averaged increment of spacing per unit of $n$ is $1.78 \AA$ for the hexagonal columnar phase, which is relatively larger than $0.98 \AA$ of the layered phase.

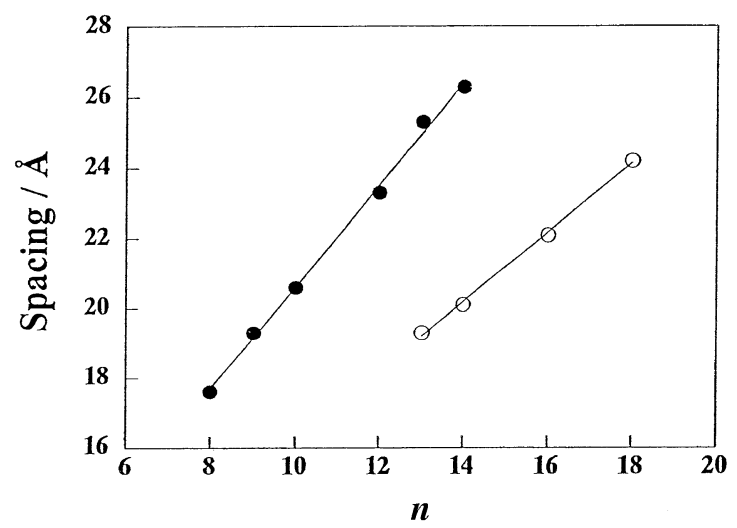

Figure 4. Variation of the 100 spacings for the layered mesophase (open circles) and the columnar mesophase (closed circles) with $n$, the number of carbon atoms in the alkoxy side chain.

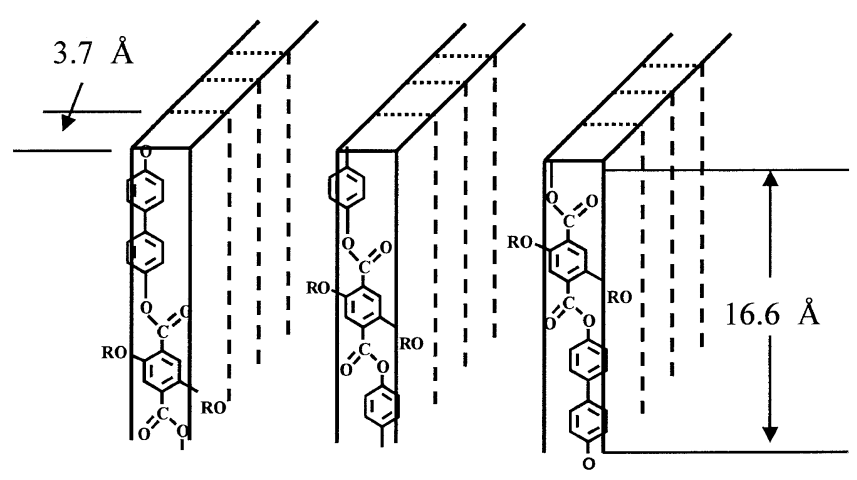

Figure 5. Schematic illustration of the structure for the layer mesophase. Here, the fully extended main chains are packed into a layer with a lateral spacing of $3.7 \AA$ and the side chains in the melt occupy the space between the layers.

Table III. Parameters in the layered mesophase

\begin{tabular}{lccc}
\hline \multirow{2}{*}{ Sample } & $\begin{array}{c}\text { Layer spacing } \\
\mathrm{d}_{100} / \AA\end{array}$ & $\begin{array}{c}\text { Calculated spacing } \\
\text { between main chains } \\
\text { within a layer } / \AA\end{array}$ & $\begin{array}{c}\text { Observed } \\
\text { density }\end{array}$ \\
\cline { 3 - 4 } & 19.3 & 3.45 & 1.07 \\
\hline PBpT-O13 & $19.1)$ \\
PBpT-O14 & 20.1 & 3.48 & 1.06 \\
PBpT-O16 & 22.1 & 3.47 & 1.04 \\
PBpT-O18 & 24.2 & 3.45 & 1.02 \\
\hline a'Calculated from the $\mathrm{d}_{100}$ spacing and observed density.
\end{tabular}

${ }^{\mathrm{a}}$ Calculated from the $\mathrm{d}_{100}$ spacing and observed density.

\section{DISCUSSION}

We first refer to the layered structure which are formed from the polymers with $n=13$ to 18 . In Figure 5 , the layered phase is schematically illustrated. The aromatic main chains construct the layer and the aliphatic side chains are spaced between layers. Each aromatic chain is extended with the repeating length of 16.6 $\AA$. The distance between the main chains within each layer that can be calculated from the layer spacing and density, is around $3.5 \AA$ as shown in Table III. Thus, the outer broad reflection with a spacing of $3.7 \AA$ that is 
Table IV. Parameters in the hexagonal columnar phase

\begin{tabular}{|c|c|c|c|c|c|}
\hline \multirow{2}{*}{ Sample } & \multirow{2}{*}{$\begin{array}{c}\text { Hexagonal lattice } \\
\text { parameter } \\
\mathrm{a} / \AA\end{array}$} & \multirow{2}{*}{$\begin{array}{c}\begin{array}{c}\text { Observed } \\
\text { density }\end{array} \\
\mathrm{g} \mathrm{mL}^{-1}\end{array}$} & \multirow{2}{*}{$\begin{array}{l}\text { Number of chains } \\
\text { included in a unit } \\
\text { cell } \quad m\end{array}$} & \multicolumn{2}{|c|}{$\begin{array}{l}\text { Distance between the neighboring } \\
\text { main chains/ } \AA\end{array}$} \\
\hline & & & & Model $\mathrm{A}^{\mathrm{a}}$ & Model $\mathrm{B}^{\mathrm{b}}$ \\
\hline PBpT-O8 & 20.4 & 1.11 & 7.0 & 6.8 & 4.4 \\
\hline РBpT-O9 & 22.3 & 1.10 & 7.9 & 6.6 & 4.3 \\
\hline PBpT-O10 & 23.8 & 1.09 & 8.5 & 6.5 & 4.4 \\
\hline PBpT-O12 & 26.8 & 1.07 & 9.7 & 6.4 & 4.3 \\
\hline PBpT-O13 & 29.2 & 1.07 & 11.1 & 6.1 & 4.2 \\
\hline РВрТ-O14 & 30.5 & 1.06 & 11.5 & 6.2 & 4.2 \\
\hline
\end{tabular}

${ }^{\mathrm{a}}$ Calculated from the equation, $(\sqrt{3} \pi)^{\frac{1}{2}} a / m$. ${ }^{\mathrm{b}}$ Calculated from the equation, $\sqrt{3} a /(m+1)$.

observed on the equatorial line, is reasonably assigned to the lateral packing of main chains within a layer.

Taking the extrapolation of the spacing data shown in Figure 4 to $n=0$ gives the average bulkiness of the backbone to be $7.0 \AA$. From this value, the densities of main-chain and side-chain domains are simply calculated as $1.34 \mathrm{~g} \mathrm{~mL}^{-1}$ and $0.80-0.83 \mathrm{~g} \mathrm{~mL}^{-1}$, respectively. The similar density of side-chain domain $\left(0.83 \mathrm{~g} \mathrm{~mL}^{-1}\right)$ is also estimated from the increment of $\mathrm{d}_{100}$ from $19.3 \AA$ to $24.2 \AA$ with the increase of $n$ from 13 to 18 . Thus, the layer structure is characterized by the high contrast in the density between the main-chain and side-chain domains.

We next consider the hexagonal columnar phase. Since each chain is also extended to have the repeating length of $16.6 \AA$, the number of polymer chains included in a hexagonal lattice is elucidated from the lattice parameter and observed density in the fourth column of Table IV. Interestingly, it increases from 7 to 12 with the increase of carbon number of side chains from 8 to 14 . No integer number means that it is just an averaged value; in some part the number of chains in a unit cell may be different from that in other part. Such a situation is allowed not for the crystal, but for the mesophase.

Here, we can propose two types of hexagonal columnar phases in Figures $6 \mathrm{a}$ and $6 \mathrm{~b}$. Both structures basically consist of the microsegregation of the aromatic main chains and aliphatic side chains as in the layered phase. In model $\mathrm{A}$, the main chains segregated from the side-chain microdomains construct the isolated columns which are packed into a hexagonal lattice (see Figure 6a). In model B, the main chains form the honeycombed network to surround the cylindrical microdomain of side chains (Figure 6b). In each model, the distance between main chains is calculated from the lattice parameter and number of chains included in a unit cell and listed in fifth column of Table IV. In model $\mathrm{A}$, the values are around $6.4 \AA$ while those for model B are around $4.3 \AA$. The former value is considered to be too large for the molecular distance while the latter is

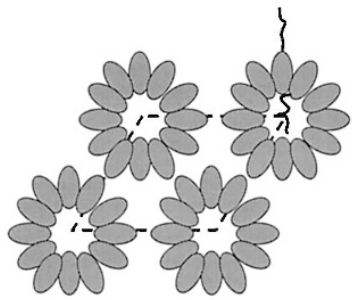

(a)

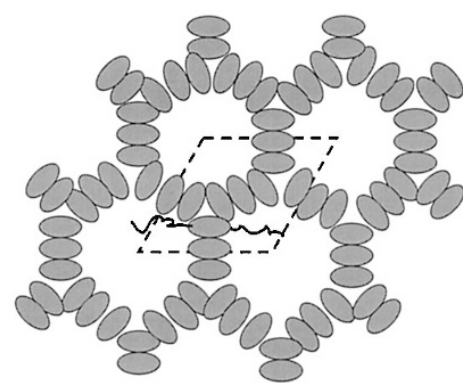

(b)
Figure 6. Two types of hexagonal columnar phases supposed on the basis of the segregation of the aromatic main chains (closed ellipsoids) and aliphatic side chains into a layer form as in Figure 5. In (a), the aromatic main chains form the closed cylinder with the side chains occupying the spaces outside and inside of the cylinders (model A). In (b), the main chains form a honeycombed network and the molten side chains are spaced within a cylindrical domain (model B).

reasonable and corresponds to the spacing of the outer broad reflection observed on the equator. Thus, model $\mathrm{B}$ is reasonably selected as reported previously. ${ }^{17}$ The increment of spacing with $n$ shown in Figure 4 gives the density of $0.91 \mathrm{~g} \mathrm{~mL}^{-1}$ for side chain domains. This value is also reasonable for the molten alkyl side chains although it is somewhat larger than that in the layered phase.

The simple geometrical constraints that are related to the ratio of the main-chain volume to side-chain volume can explain the transformation of layered phase to columnar phase with a decrease of the carbon number of side chain. In this case, the main chain volume is constant and so the ratio increases with a decrease of the carbon number in side chain. Thus, the increased ratio requires the truncated cone shape for each repeating unit, ${ }^{23}$ leading to cylindrical microsegregation. In this limited polymer system, the critical ratio below which the columnar phase is favored is around 0.44. It is interesting to note that on the same basis several types of columnar phases have been formed from the bolaamphiphiles with lateral alkyl chains. ${ }^{24}$

The coexistence of the layered and columnar phases 


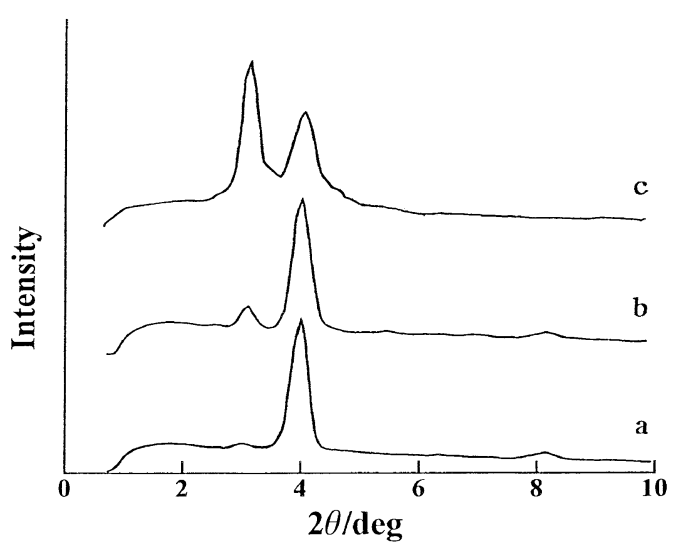

Figure 7. Intensity profile for the 100 reflections of the layered and columnar mesophases in PBpT-16 prepared with different thermal histories: (a) quenched to $100^{\circ} \mathrm{C}$ from the nematic liquid crystal at $150^{\circ} \mathrm{C}$ and annealed for $6 \mathrm{~h}$, (b) quenched to $125^{\circ} \mathrm{C}$ and annealed for $6 \mathrm{~h}$, and (c) slowly cooled to $130^{\circ} \mathrm{C}$ at a rate of $0.1^{\circ} \mathrm{C}$ $\mathrm{min}^{-1}$ and annealed for $6 \mathrm{~h}$.

in polymers with the intermediate $n$ indicates a small energy difference between them. It should be noted here that the relative amount of the phases depends on the molecular weight. For example in PBpT-O12 polymers, the columnar phase is stably formed from the higher molecular weight sample with the inherent viscosity larger than $0.3 \mathrm{dL} \mathrm{g} \mathrm{g}^{-1}$, but it becomes coexist with the layered mesophase when the inherent viscosity is smaller. ${ }^{25}$ Thus, the coexistence of the two phases is considered to be due to the wide distribution of molecular weight. In other words, it is suggested that the clear transition between the two phases would take place at a certain temperature if the polymers with narrow molecular weight distribution are prepared. Understanding of the transition behavior will be done after the detailed studies on the dependency of $M_{\mathrm{w}}$ or $M_{\mathrm{w}} / M_{\mathrm{n}}$. Irrespective of this ambiguous factor, it can be safely said that the columnar phase exists in the higher temperature region than the layered phase. Figure 7 shows the intensity profile with respect to the 100 reflections of the layered and columnar phases in PBpTO16 prepared through different thermal histories. It is obvious that the relative intensity of 100 reflection of the layered phase increases with the decrease of the mesophase temperature. Thus, we conclude that the layered phase is thermodynamically lower temperature mesophase than the columnar one.

\section{CONCLUSION}

In this study, we have prepared the homologous series of PBpT-On, poly( $p$-biphenylene terephthalate) with alkoxy side chains attached to terephthalate moiety. The thermotropic liquid crystalline phases are well formed from the PBpT-On polymers with the car- bon number of alkoxy side chains, $n$, ranging from 8 to 18 . The nematic liquid crystal is the highest temperature mesophase for all the polymers and two other mesophases, hexagonal columnar and layered mesophases, are formed in the lower temperature range. The layered mesophase with the main chains packed into a layer and the side chains spaced between the layers, is preferentially formed from the PBpT-On with $n=18$. The densities of the main chain and side chain microdomains within a layer were elucidated as 1.34 and $0.83 \mathrm{~g} \mathrm{~mL}^{-1}$, respectively. For the PBpT-On with the smaller $n=8-12$, the lower temperature mesophase was found to be a hexagonal columnar phase. In PBpTOn with the intermediate $n=13,14$, and 16, the columnar phase is also formed, but coexists with the layered mesophase. Interesting is the fact that many chains are included in a hexagonal unit cell of the columnar phase. The number of chains is increased from 7 to 12 with the increase of $n$ from 8 to 14 . This unusual feature was satisfied by only the special hexagonal packing structure where the fully extended main chains are closely packed with the lateral spacing of around $4.5 \AA$ to form the honeycombed network and the molten side chains are accommodated within resulting cylindrical domains. In PBpT-O16, it was found that the relative amount of the columnar phase to layered phase increases with the increasing temperature, leading to the conclusion that the columnar phase is thermodynamically higher temperature mesophase than the layered one.

\section{REFERENCES}

1. J. Watanabe, H. Ono, I. Uematsu, and A. Abe, Macromolecules, 18, 2141 (1985).

2. M. Ballauff, Angew. Chem., Int. Ed. Eng., 28, 253 (1987).

3. M. Ballauff and G. F. Schmidt, Mol. Cryst. Liq. Cryst., 147, 163 (1987).

4. J. M. Rodrigues-Parada, R. Duran, and G. Wegner, Macromolecules, 22, 2507 (1989).

5. M. Ebert, O. Herrmann-Schenherr, J. Wendorf, H. Ringsdorf, and P. Tschirner, Liq. Cryst., 11, 249 (1990).

6. A. Adam and H. W. Spiess, Makromol. Chem., Rapid Commun., 11, 249 (1990).

7. R. Stern, M. Ballauff, G. Lieser, and G. Wegner, Polymer, 32, 2079 (1991).

8. B. R. Harkness and J. Watanabe, Macromolecules, 24, 6759 (1991).

9. J. Watanabe, B. R. Harkness, and M. Sone, Polym. J., 24, 1119 (1992).

10. K. Marz, P. Lindner, J. Urban, M. Ballauff, and E. W. Fischer, Acta Polym., 44, 139 (1993).

11. S. B. Damman and G. J. Vroege, Polymer, 34, 2773 (1993).

12. J. Watanabe, B. R. Harkness, M. Sone, and H. Ichimura, Macromolecules, 27, 507 (1994). 
13. M. Sone, B. R. Harkness, H. Kurosu, I. Ando, and J. Watanabe, Macromolecules, 27, 2769 (1994).

14. K. Fu, T. Nematsu, M. Sone, T. Itoh, T. Hayakawa, M. Ueda, and J. Watanabe, Macromolecules, 33, 8367 (2000).

15. J. Watanabe, H. Kamee, and M. Hujiki, Polym. J., 33, 495 (2001).

16. K. Okoshi, H. Kamee, G. Suzaki, M. Tokita, M. Fujiki, and J. Watanabe, Macromolecules, (in press).

17. J. Watanabe, N. Sekine, T. Nematsu, M. Sone, and H. R. Kricheldorf, Macromolecules, 29, 4816 (1996).

18. J. Watanabe and Y. Takashina, Macromolecules, 24, 3423 (1991).
19. T. Yamagishi, T. Fukuda, T. Miyamoto, Y. Yakoh, Y Takashina, and J. Watanabe, Liq. Cryst., 10, 467 (1991).

20. G. Unger, Polymer, 34, 2050 (1993).

21. A. Takada, K. Fujii, J. Watanabe, T. Fukuda, and T. Miyamoto, Macromolecules, 27, 1651 (1994).

22. K. Suehiro, Y. Chatani, and H. Tadokoro, Polym. J., 7, 352 (1975).

23. G. E. Molau, "Block Polymers", S. L. Aggarwal, Ed., Plenum Publishing Corporation, New York, N.Y., 1970.

24. C. Tshierske, J. Mater. Chem., 11, 2647 (2001).

25. K. Fu and J. Watanabe, to be published. 